\title{
Organisational Change to Health Promoting Hospitals: A Review of the Literature
}

\author{
Chiachi Bonnie Lee $\cdot$ Michael S. Chen • \\ Michael John Powell · Cordia Ming-Yeuk Chu
}

Received: 17 March 2013/Revised: 13 June 2013/Accepted: 20 June 2013/Published online: 10 July 2013

(C) The Author(s) 2013. This article is published with open access at Springerlink.com

\begin{abstract}
The Health Promoting Hospitals (HPH) initiative, as a setting approach, was launched by the World Health Organisation in 1988, and widespread expansion and development throughout the world ensued. This paper elaborates on and clarifies the concept of HPH and highlights the development of health-promoting settings in hospitals. This review also examines the enabling and hindering roles of organisational factors in reorienting hospitals towards health-promoting settings. This paper reaffirms the significance of organisational change in building capacity for health promotion during the development of HPH and notes that hospitals require systematic organisational support to fulfil their roles in promoting population health. Nevertheless, this review suggests that many of the identified barriers are related to insufficient organisational support. In particular, the low prioritisation of health promotion in hospital missions, shortages of resources, ineffective project management, lack of communication, poor coordination and integration and inappropriate job-person matches were six major reported barriers. Organisational capacity building for health promotion must be considered if hospitals are to adopt the HPH initiative.
\end{abstract}

Keywords Health Promoting Hospitals . Organisational change $\cdot$ Capacity building

C. B. Lee $(\bowtie) \cdot$ M. S. Chen

National Chung Cheng University, Chia-yi County, Taiwan, Republic of China

e-mail: bonnie1012@mail2000.com.tw

M. J. Powell · C. M.-Y. Chu

Griffith University, Brisbane, Queensland, Australia

\section{Introduction}

Many prominent international entities have called for integration of health service and health promotion in response to challenges to health systems, such as the need for a reduction in health care costs and for the effective prevention and management of non-communicable diseases [58-61]. Notably, among these entities, the World Health Organisation (WHO) launched the Health Promoting Hospitals (HPH) project in Europe in 1988, leading to widespread expansion and development throughout the world. The European Pilot Hospital Project has demonstrated the feasibility and applicability of HPH in all types and sizes of hospitals in widely diverse health systems [47]. By May 2011, HPH comprised 39 networks in 26 countries on five continents, including individual hospitals that do not belong to a national/regional network, and HPH has 841 members across 51 countries [45]. The HPH project is a form of setting-based health promotion and aims to 'improve health gain for its stakeholders by developing structure, cultures, decisions and process' [57], p. 6).

However, there is an 'old wine in the new bottle' doubt in that hospitals have used the HPH project as a banner but have not built a supportive organisational infrastructure for health promotion [21]. It has been well recognised that capacity building is key to effective health promotion practices [27, 61]. Organisational change is regarded as an effective way to build capacity for health promotion $[18$, 23]. The effect of organisational capacity building for health promotion extends beyond theory and could multiply health gains [16], achieve program sustainability [63], ensure the quality assurance of health services $[33,39,50]$ and yield more health promotion activities and strategies [31, 44]. McHugh et al. [33] have examined the literature on HPH or health promotion (HP) programs and have 
found a dearth of evaluative studies in this area. Subsequent research was published successively $[19,30,31$, $35,54]$. This study reviews the development of this concept and its implementation so far, focusing on the enabling and hindering roles of organisational factors in reorienting hospitals towards health-promoting settings.

\section{Background}

The Declaration of Alma Ata of 1978 argues that primary health care should be the kingpin in achieving population health [59]. In 1986, one of the five main health promotion actions identified in the Ottawa Charter was to reorient health services towards health-promoting settings, which was incorporated in the First Global Conference on Health Promotion [60]. In 2009, the Nairobi Call to Action was highlighted in the 7th Global Conference on Health Promotion, again stressing the importance of strengthening health systems [61]. Furthermore, the European Health Report 2009 proposed that health services and health outcomes could be enhanced through health promotion action [58].

Several factors explain the leading role of hospitals in implementing health promotion: hospitals are central to the health care system $[7,15]$ because they consume 40-70\% of the national health care expenditure [11]; health promotion can encourage hospitals to improve the quality of health services $[11,38]$; a hospitalised person is conveniently exposed to health promotion information and can be aptly persuaded to change unhealthy behaviour [48]; hospitals function as a workplace, health care centre and community institute, reaching a large number of people [15]; workplace health promotion in hospitals can help to address the health-related problems of workers [15, 46], such as short-term absenteeism, thereby improving individual and organisational capacity; and medical professionals can make a greater impact on public policy due to their role in medical practice and social education, and people are likely to accept suggestions from these professionals [15, 46]. Last, but perhaps of the most pressing nature, hospitals produce a large amount of waste and are also consumers of many products $[8,11]$. Hospitals should assume the corporate responsibility of reducing the amount of waste and should purchase 'green' products that are beneficial to both the environment and human health. Therefore, hospitals have potential important roles in promoting population health by developing healthy settings.

\section{The Concept: HPH as a Setting Approach}

As the Declaration of Alma Ata, the Ottawa Charter, and many other important international documents have suggested, health promotion is hardly a new concept. However, hospitals might have implemented health promotion only in a narrow way. In the 1980s, a large number of hospitals in North America, Europe, Australia and New Zealand implemented health promotion programs [24]. Nevertheless, most of these programs have been limited to education, behavioural change on the part of individuals and health screening for disease by individuals and professionals, often on an ad hoc basis [14, 20, 24, 26]. Furthermore, health promotion programs were conducted as isolated projects or as an assignment to a particular division, without back-up organisational commitment [21].

The narrow role of such hospitals in health promotion can be observed, in contrast to the typology proposed by Johnson and Baum [21]. Based on two criteria-the extent to which hospital organisation is involved and the types of health promotion activities that are performed - the authors proposed four types of HPH from an organisational perspective. Type one is doing a health promotion project. This approach does not confront disease-based practices to any significant extent or reorient the overall hospital organisation and staff members' roles towards health promotion. Health promotion projects are implemented on an ad hoc basis, which has little to do with organisational strategic development. Type two is delegating health promotion to the role of a specific division, department or staff, such as a health promotion coordinator or the Department of Community Health. This approach does not necessarily confront each unit within the hospital to force a change in the unit's focus away from medicalisation and institutionalisation and towards health and community orientation. This approach can have only a limited impact, as the approach might alienate the staff of the departments that were not assigned the responsibility of health promotion. Type three is being a health promotion setting, the dominant approach recognised and advocated by the International HPH Network. This approach calls for hospitals' commitment to organisational change as a way to create health-promoting settings, and in turn, the hospital can undertake health promotion activities to promote the health of patients, the staff, the organisation and its physical environment. This approach falls short of bringing community health into the picture. Last, type four is being a health promotion setting and improving the health of the community. Using this approach, hospitals are committed not only to turning themselves into health-promoting settings but also to improving the health of the community. The health issues of patients, the staff, the organisation, the physical environment and the community are all taken care of through the implementation of HPH without walls. Of these four types, Johnson and Baum [21] argue that only the last two types can be identified as genuine HPH approaches. 
Several authors have criticised the narrow views of health promotion in hospitals that focus on education, the behavioural change of individuals, risk and problem orientation, the prevention of disease occurrence and the implementation of these activities only through ad hoc projects or a specific person or department, without much organisational back-up [15, 24, 21, 51, 42]. In contrast to these narrow views of HP, broader views of HPH aim to systematically utilise and perform organisational functions and resources to build health-promoting settings, with the goal of maintaining and improving not only the ill health but also the good health of stakeholders [41, 57]. This perspective on HP is not limited to the primary prevention of diseases but also extends to secondary and tertiary prevention $[43,51]$. The perspective is distinct from the traditional conception proposed by Leavell and Clark [28], who maintained a model of three-level preventive measures against diseases in which HP is only used to prevent diseases. Therefore, to encourage more medical professionals who are accustomed to treating symptoms after disease occurrence to participate in $\mathrm{HPH}$, it is necessary to provide more information about and stress the significance of the broad concept of HPH.

\section{Application of the Burke-Litwin Causal Model}

The effective reorientation of health services calls for the commitment of hospitals to change into health-promoting settings with corresponding organisational input [21]. Organisational change management builds on the diagnosis of a need for change [4, 6, 17]. Hayes [17] argues that organisational models can determine what type of information requires attention. These models explain what has been observed in the organisation and what action should be taken. The organisational model is defined as 'a representation, to show the construction or appearance of organisation' [4]. Currently, many organisational models are available, such as Leavitt's Organisational Systems Model [29], Weisbord's Six-Box Model [52], Nalder-Tushman's Congruence Model [34], Kotter's Integrative Model of Organisational Dynamics [25], Tichy's Technical, Political, Cultural Framework [49] and Burke-Litwin's Causal Model of Organisational Performance and Change (the Burke-Litwin Causal Model hereafter for short) [5]. The latter model, incorporates a comprehensive set of factors, is based on the experiences and logics from practical fields [5] and can be used as a useful framework for reviewing health promotion action through an organisation [23].

The Burke-Litwin Causal Model is based on an opensystem principle in that the model assumes that an organisation is responsive to a shift in the external environment. The model is constructed from transformational factors and transactional factors [4, 5]. Transformational factors refer to the external environment, leadership, mission and strategy and organisation culture. These factors are very sensitive to outside environmental dynamics and also demand new input from the entire organisation. These changes exert considerable influence over the entire organisation. In contrast, transactional factors refer to daily routine operations at the group and individual levels. These factors encompass structure, management practices, systems, work unit climate, task requirements, individual skills and ability, individual needs and values, motivation; and individual, group and organisational performance.

\section{Health Promotion Action Through the Organisation}

The rest of this article will be mainly devoted to the application of the Burke-Litwin Causal Model in HPH implementation. In light of current $\mathrm{HPH}$ research and related literature, this section will elaborate on the enabling and hindering roles of the organisational factors in the BurkeLitwin Causal Model in reorienting hospitals towards healthpromoting settings.

The Transformational Factors

\section{External Environments}

Hospital management is closely linked to external environmental dynamics. Exterior environments can facilitate or hinder the hospital's ability to perform health promotion. The experience of European hospitals in the implementation of $\mathrm{HPH}$ project illustrates the positive impact of the external environment on HPH. In the 1990s, external forces, including the need for cost reduction, new market direction and the increasing demands and expectation of the population, drove hospital reforms and subsequently the move towards HPH [8]. NHS Health Scotland formulated various national policies and regulations that built a supportive context for the implementation of Health Promoting Health Services (HPHS) by assigning HPHS 'Co-ordinators' or 'Leads' who undertook HPHS work and played supportive roles in consultation and resource linkages [35].

In contrast, external environmental conditions can also hinder hospitals from performing health promotion. A lack of national and regional leadership and health policy commitment may hamper hospitals' efforts to integrate health promotion into daily practices $[20,53]$. In China, Beijing's experience illustrates this concept well. The reimbursement system of the health insurance scheme does not include services for health education and health promotion in Beijing. In other words, health education and health promotion do not generate hospital income; therefore, hospital leaders 
tend to ignore these activities [13]. The same barrier has also existed in southern Australia [20]. Additionally, NHS Health Scotland [35] found that there was a loose link between HPHS structures and existing relevant initiatives at the national level, such as the Community Planning Agenda and Single Outcome Agreements.

In sum, the implementation of HPH programs requires a supportive external context for HPH to increase hospitals' motivation to adopt and apply this concept. A supportive external context is exemplified by HPH-related policies or regulations, available operational support and the reimbursement system of the health insurance scheme integrated with health promotion. Without incentives from external environments, hospitals might be reluctant to implement health promotion.

\section{Leadership}

To sustain health promotion in hospitals, the role of leadership is essential. Leadership can fulfil the potential of HPH. The Comprehensive HPH Approach, involving both project management and organisational development, requires the health promotion involvement of leadership [43]. The Integration Model, incorporating both health promotion and quality management and serving as a WHO self-assessment tool for health promotion in hospitals [9], also indicates the significance of leadership.

The contributing role of leadership in the process of health promotion in hospitals has been investigated in recent studies [13, 20, 35, 39]. However, in reality, leaders might not consider preventive and health-promoting efforts to be a first priority, as a case study in Sweden has documented [19]. Present studies also show that managers may not be enthusiastic about performing health promotion, partly because these individuals do not have an adequate understanding of the concept of HPH [13,30]. Tountas et al. [50] report that one of the barriers that arose in the development of an HPH program in Aretaieion Hospital in Greece was the lack of background in developing health promotion and suggested that it is essential for the hospital organisation to adopt the principles of HPH publically and officially. The people who are in the best position to implement an $\mathrm{HPH}$ program are the hospital leaders. In contrast to the Greek experience, a good example of success in HPH is the Rudolfstiftung Hospital in Austria, which has earned support from hospital owners and management through the process of the Vienna WHOModel Project [37].

\section{Mission and Strategy}

Mission signifies the focus of an organisation and can be achieved through planned strategies. The enabling roles of mission and strategy in HPH have been elaborated in several theories. The Comprehensive HPH Approach requires that hospitals' mission statements embrace health promotion as an explicit aim and value and that hospitals also develop health promotion strategic policy documents with the details of aims, goals, targets and health promotion principles and core strategies and policies as a guide to implementation [43]. In the Integration Model, the WHO HPH manual and self-assessment form [9] also demonstrate the key roles of mission and strategy in HPH. For instance, Standard 1.1.1 states that the hospital's stated aims and mission include health promotion. Johnson and Paton [23] propose a series of questions to reflect on whether the hospital's mission and strategy are supportive of health-promoting health services. For example, is there a health promotion strategy that includes integration into practice and population health priorities?

With reference to positive influences, Johansson et al. [19] state that one of the possibilities for a more healthpromoting health service is to make health promotion a prioritised assignment (p. 3). Pelikan [40] argues that to fulfil the potential of the Comprehensive HPH Approach, hospitals have to integrate health promotion values and principles, goals and targets, standards, criteria and indicators into (their) written vision, mission statement... (p. 267). A written mission statement incorporating relevant health promotion principles is regarded as the starting point for hospitals to prioritise health promotion.

However, several barriers related to the implicit mission of health promotion have been identified in recent studies. Tountas et al. [50] find that one of the barriers to the development of an HPH program in Aretaieion Hospital in Greece was a failure to design an explicit policy of health promotion and suggested that it is imperative for the hospital organisation to adopt the principles of HPH. The mission could be presented in a written mission statement including health promotion principles. Recent studies have also found a common difficulty in the lack of prioritisation of health promotion [19, 20, 53]. Regarding achievements, the Rudolfstiftung Hospital in Austria succeeded in integrating the main goals of HPH into the mission statement of the hospital through the process of the Vienna WHOModel Project [37].

After formulation of the mission statement, the next important step is to develop a strategy to implement the mission. Hospitals could implement health promotion based on the Addition Model, the Integration Model [3], seven health promotion quality management strategies [40] or manual and self-assessment forms for implementing health promotion in hospitals [9]. In terms of selection between the Addition Model and the Integration Model, Pelikan in his address in Brisbane (at the Workshop on Concepts and Application of Health Promoting Settings held in December 2009 by Centre for Environment and Population Health of 
Griffith University, Brisbane, Australia) suggests that hospitals that do not have a well-developed quality management system should adopt the Addition Model; otherwise, the hospitals should integrate quality management with health promotion by adopting the Integration Model. An explicated strategy plays a key role in supporting an organisation's implementation of an HPH program.

\section{Organisational Culture}

Organisational culture is systematically presented in the form of artefacts, norms, values and basic assumptions [6]. The change from a medical organisational culture to a health-promoting organisational culture can be achieved by a change in such forms. The enabling roles of organisational culture in HPH are exemplified and demonstrated in many recent studies. Cultural change is difficult because it demands change in fundamental and time-honoured areas of an organisation. Johnson [20] builds a case study on a hospital in southern Australia and argues that to reorient health services, there is no need to change the attitudes and behaviours of all staff members but only those of the key leaders and managers. Johnson further explains that with organisational support, key people who have a good understanding of HPH can successfully implement strategic health-promoting activities for health services [20].

In reality, hospitals seeking to adopt an HPH program frequently encounter barriers related to an unsupportive organisational culture. Previous studies have shown that hospitals still hold an attitude that tilts towards risk factors, disease treatment and quantifiable services, and accordingly, little attention is given to the holistic and salutogenic perspective on health and resources [19, 20, 22]. Moreover, Johansson et al. [19] also note that the hospital system in Sweden does not value the staff's wellbeing and strengths and argue that this situation should be improved because improvement will in turn have a positive influence on patients [19]. Experience in Scotland has also suggested that HPHS was impeded by a hospital organisational culture that did not value health improvement tasks [35].

\section{The Transactional Factors}

\section{Structure}

Organisational structure refers to the way in which the overall work of an organisation is divided into subunits and the way in which these subunits are coordinated to complete tasks [6]. To accomplish a comprehensive HPH approach, health-promoting structures are required, and the soundness of such a structure can be evaluated against the following criteria [43], p. 66): specific health promotion management structure; health promotion steering committee (including a member of the directorate of the hospital); health promotion manager/team (reporting directly to directorate of hospital); network of health promotion focal points in all sub-units of hospital.

In the Integration Model, the WHO-HPH manual and self-assessment form [9] also demonstrate the key roles of structure. For instance, Standard 1.1.4 states that the hospital identifies personnel and functions for the coordination of HP. In addition, Johnson and Paton [23] have designed a series of questions to reflect on whether the hospital's organisational structure is supportive of health promotion, such as 'where does health promotion fit within the structure of the organisation?'.

A number of positive influences of structure on HPH have been identified in many recent studies. Johnson and Nolan [22] argue that it is important for hospitals to construct a formal structure of role agreement for management cooperation programs with community organisations despite existing informal networking opportunities. Nowak et al. [36] highlight several of the supportive structures established in the Rudolfstiftung Hospital in Vienna (1998, p. 65):

- Identify someone or a group of persons in the hospital who can be the coordinating and leading person/group for an ongoing process and appoint a project coordinator

- Establish an inter-professional committee for developing and deciding the Health Promotion strategy, including the management of all professional groups, staff counsel and health promotion experts

- Establish project groups for each problem area which can represent all different perspectives for solving these problems

- Establish clear roles and co-operation structures within the project groups and for the co-operation of persons and institutions

Despite these measures, hospitals have encountered barriers related to organisational structure while implementing an HPH program. Johnson and Baum [21] comment that health promotion activities are rarely performed throughout an entire organisation but are rather restricted to an isolated and incomplete project or are made the responsibility of an individual staff member. The reason might be related to the lack of hospital structures that support the integration of health promotion into entire hospital structures [56]. To overcome this problem, it has been suggested that the HPH initiative could be integrated into a quality management structure, such as the European Foundation for Quality Management (EFQM) Model and Balanced Scorecard [3, 10, 40]. Brandt and other scholars [3] find further appeal in the greater involvement of directors, as in the EFQM Model, to render the integration of health promotion into quality management effective. In 
the Addition Model, Johnson [20] finds that an organisational structure with a centralised decision-making process hindered the development of department-oriented initiatives because the department directors could not find financial resources or lead the department strategically. Health promotion relies on multidisciplinary work; however, it is a challenge to develop strong coordination between departments in the hospital. Johnson [20] finds that multidisciplinary teams often experience high levels of conflict. Recent research finds that this challenge might originate from the lack of a specialised department to take the lead by coordinating hospital promotion-related human resources [30].

This lack of cooperation is a significant issue because Standard 5 of the WHO HPH Standards highlights the importance of continuity and cooperation through a planned approach to ongoing collaboration between hospitals and other health service stakeholders [9]. However, Johnson and Nolan [22] find that a barrier to cooperation between the hospital and community organisations may be due to a lack of clear and firm links between the hospital and the community.

Recent studies have demonstrated the important roles of organisational structures in reorienting health services. From the perspective of capacity building, hospitals should consider building supportive structures prior to the implementation of an HPH program. In the case of the Rudolfstiftung Hospital in Austria, new professional roles and communication structures were established through the process of the Vienna WHO-Model Project, which then became an embedded force to sustain the health promotion projects [37].

\section{Management Practices}

Management practices refer to managers' regular and daily activities related to the arrangement of human and material resources to implement their organisation's strategy [5]. In particular, management practices need to focus on four areas: the first concerns managing projects $[9,21,22,35$, $40,43]$, the second is enhancing the visibility of health promotion across the organisation [3, 20, 35, 40, 43, 55], the third concerns developing partnerships or collaborations between hospitals and other health service providers $[9,22,43]$, and the fourth is encouraging staff members to take an inventive approach to tasks and projects $[19,20]$.

Regarding project management, both the Addition Model and the Integration Model highlight the significant role of project management in the implementation of HPH projects. In the Addition Model, the WHO Regional Office for Europe highlights the importance of project management in the comprehensive HPH approach and indicates the following essential criteria [43], pp. 65-66).
- Regularly monitoring, evaluating, reporting and improving initiatives of health promotion outcomes and impact (by surveys, balanced score card, reporting)

- Regularly conducting health promotion projects for planning

- Implementation of specific health promotion policies

- There have to be explicit goals, criteria, standards and indicators for health promotion outcomes for health promotion processes, for health promotion structures and for health promotion quality monitoring so that the fulfillment of being a HPH can be regularly observed, monitored, documented, evaluated, reported and improved.

In the Integration Model, the WHO HPH manual and self-assessment form also note the role of management practices in implementing health promotion in hospitals [9]. For instance, Standard 1.3.1 states that data are routinely captured on HP interventions and available to staff for evaluation.

When managers focus on improving the visibility of health promotion, Whitelaw et al. [55] argue that it is important to nurture leadership advocacy to win the attention of the current leaders and to receive the leaders' support for the HPH initiative and to foster a supportive base of committed people within the hospital organisation to enhance the visibility of health promotion across the organisation. Regarding the development of partnerships or collaborations between hospitals and other health service providers, Johnson and Nolan [22] propose that these relationships could be achieved by establishing connections between clinical and executive staff members at all organisational levels. The WHO requires that hospitals regularly participate in healthy alliances and partnerships with community partners [43].

Management practices also need to consider how to encourage staff members to take an inventive approach to tasks and projects [5]. Johansson et al. [19] report that hospitals should support reflection and learning, explore and make the best use of the competencies of existing health promotion staff members and grant the staff freedom of action. Johnson [20] considers this participatory process to be a key element. For instance, in a case study using an action research process based on a hospital in southern Australia, the hospital draws staff members who are interested in health promotion into action-based teams and allows the staff to identify health promotion activities and to include others from outside of the organisation to reorient the hospital to be more health promoting [20]. Whitelaw et al. [55] draw managers' attention to the need to develop a specific practical context in which staff members can appreciate the value of the framework applied in the HPHS; without this awareness, the framework cannot be used to its full potential. 
In contrast to examples of the facilitating role of management practices, recent studies have also highlighted barriers to management practices in project management $[1,2,10,11,19,30,50]$, in the coordination of tool dissemination $[35,55]$, in the visibility of health promotion activities [20, 30, 50], in collaboration [22], in communication [10] and in the utilisation of the existing competencies of the staff [19]. Several of the reasons that these barriers arise can be summarised as follows.

First, inadequate planning of project management is a common problem [2, 50]. Groene and other experts [10] find that the integration model, which requires the integration of health promotion into quality management, can be hindered by weak central project management. Barriers to project management include insufficient reference to theoretical foundations [2]; the implicit implication of health inequalities [35]; implicitly defined aims and priorities for health promotion [50]; inadequate attention paid to the health (including mental and social health) of the staff and organisation [30]; insufficient consideration of structural or cultural determinants [11] and a lack of evaluation methods, including a lack of appropriate indicators $[1,30]$.

Second, regarding a lack of coordination, Whitelaw et al. [55] find that the main barriers arise from the uncoordinated dissemination of a tool or resource despite the framework being in place. NHS Scotland Health [35] reviewed the Health Promoting Health Service Funded Sites and found possible overlap and duplication of activities between health promotion and health improvement initiatives and therefore recommended further coordination and better management of these two areas for more appropriate prioritization and more effective synergy.

Third, regarding the invisibility of health promotion activities, this problem results from inadequate information circulated to staff members about health promotion activities [30, 50] and inadequate communication [30]. Tountas et al. [50] argue that there is unclear communication of the rather intangible concept of 'health promotion'. Similarly, Johnson [20] believes that too much attention is paid to stories about 'miraculous' disease treatment rather than to educational and health promotion issues in hospital newsletters [20].

Fourth, regarding barriers to collaboration, Johnson and Nolan [22] indicate that collaboration between the hospital and community stakeholders is not initiated until funding is acquired, which prevents the hospital and community stakeholders from collaborative planning to identify and address health problems in communities.

Fifth, regarding insufficient communication, Groene et al. [10] report that the use of the Balanced Scorecard made it difficult to persuade the clinical staff to recognise the advantage over clinical results, i.e. it was difficult to persuade the clinical staff to accept the outcomes indicated by the Balanced Scorecard. The staff preferred to rely on clinical results. Finally, Johansson et al. [19] suggest that managers do not take full advantage of the existing health promotion competencies of hospital staff.

In addition to the barriers mentioned above, the need for the further implementation of health promotion in hospitals in terms of management practices has been analysed in existing studies. The practices can be categorised into three areas: project management, partnership and mediation. Regarding project management, Lin et al. [30] make an appeal for diligent attention to a needs assessment of stakeholders, objective setting, action plans and monitoring of the quality and effectiveness of health promotion programs. Regarding partnership, Wise and Nutbeam [62] propose that the management pursue effective ways to work with several different groups: with the community, to mobilise support for change; with the media, to affect the broader public perception about HPH and with like-minded advocacy organisations, such as the International Union for Health Promotion and Education (IUHPE), to translate knowledge and experience into fieldwork. Moreover, Johnson and Nolan [22] stress the importance of collaborative planning between the hospital and community organisations at the beginning, when projects are applied to identify and respond to health problems in the community. It is also important to develop informal and formal connections between the hospital and other external stakeholders at all levels and to develop agreements to guarantee trust before building working relationships. Last, regarding mediation, Groene et al. [10] argue that the executive of the hospital should make considerable efforts in mediation between hospital and departmental objectives.

Summarising the achievements of management practices in implementing HPH initiatives, Johnson [20] finds that throughout an HPH program, the hospital can use the results of an evaluation of community activities to lobby for changes in the state health funding policy. Additionally, in this study, hospital staff members extended the scope of health promotion targets to include patients and families, the community, the organisational and physical environment and the staff. Furthermore, the Scottish Review of Health Promoting Health Service Funded Sites found that the HPHS initiatives brought better planning; greater access to health promotion messages by the staff, patients and visitors and a shift in organisational practices [35].

\section{Systems}

Systems refers to policies, resources and procedures that can support organisation members to work in their jobs and to fulfil their roles' responsibilities $[4,5]$. In the Addition Model, the WHO Regional Office for Europe stresses the significance of systems in the Comprehensive HPH 
Approach and indicates the following criteria [43], p. 66): specific annual health promotion action plan; formulated health promotion strategic policy document, specifying aims, goals, targets and health promotion principal and core strategies and policies to reach them; specific budget ear-marked for health promotion; specific health promotion organisational manual.

In the Integration Model, the WHO HPH self-assessment tools [9] demonstrate a wide range of the supportive roles of systems in implementing health promotion in hospitals. For example, Standard 1.2.1 states that there is an identifiable budget for HP services and materials. This tool presents the importance of HP-inclusive resources, policies/guidelines and procedures. Johnson and Paton [23] highlight the importance of HP-inclusive polices, resources and subsystems when hospitals attempt to determine whether their systems are supportive of health promotion.

In addition, many recent studies have suggested that the following aspects of systems were key enablers of the implementation of HPH programs: available funding [3, $13,21,22,40,43]$, health promotion policies [20, 21, 32, 40, 43], available personnel [13, 20, 21], skilled health promotion support $[20,55]$, plans, manual, standards or frameworks for health promotion [12, 21, 43, 55], time allocation [19], access to a tool or framework [55] and available physical facilities [21]. Notably, standards or frameworks can work well only with the corresponding input of coordinated efforts and supporting infrastructure $[12,33,55]$.

In contrast, previous research identified barriers related to unsupportive policies and procedure and insufficient resources. First, a lack of resources is a key issue, including funding $[1,13,22,30,50]$, personnel $[1,13,20,22,30$, 50], time [10, 13, 19, 20, 35, 50], facilities [20, 30], space $[19,30]$, health promotion guidelines [1] and evidence of the effectiveness of health promotion [20].

Second, in terms of unsupportive policies, Tountas et al. [50] finds a problematic lack of an effective communication policy, which results in insufficient information for hospital staff members about the health promotion activities implemented by the hospital. Johnson [20] finds a problem with the hospital's implicit policy on health promotion. Last, regarding unsupportive procedures, Johnson and Nolan [22] highlight the lack of a mechanism for hospital staff at all levels to meet and establish networks with community organisations both formally and informally.

\section{Work Unit Climate}

Climate refers to the corporate perceptions generated unobtrusively and imperceptibly among staff members working within the same work departments [5]. Standard 4.2.2 of the WHO HPH manual and self-assessment form
[9] requires that staffs in all departments are aware of the content of the organisation's health promotion policy ( $\mathrm{p}$. 48), which can help build health promotion corporate perception among staff members. From the perspective of capacity building, it is important to pursue a supportive work unit climate throughout the process of the HPH initiative. Scottish experiences may shed light on this concept. In Scotland, a health promotion initiative in health services has led to increased social interaction among the staff [35].

\section{Task Requirements and Individual Skills/Abilities}

This category refers to indispensable behaviours aimed at producing maximum task effectiveness, achieved through specific skills and knowledge that are usually considered to be essential for staff members to complete the assigned work [5]. To achieve the best results from a comprehensive HPH approach, health promotion education and training for the staff and leadership are required [43]. Similarly, the WHO HPH manual and self-assessment form also require training and the development of health promotion skills among the staff. Standard 4.2.1 states that New staff receives an induction training that addresses the hospital's health promotion policy [9].

Task requirements, health promotion training and the competence of staff members have been explored and detailed in recent research [3, 13, 20, 21, 55]. Johnson [20] further proposes the application of the experiential approach to learning when hospitals host health promotion training. Moreover, Johnson and Baum [21] note the importance of integrating health promotion into job descriptions.

However, many relevant barriers have been identified in recent studies of hospitals performing health promotion. One common barrier relates to a shortage of health promotion skills. Another difficulty is that staff members often have not accepted the concept of HPH [13, 20, 32, 35, 55]. Guo et al. [13] report that hospital managerial staff often has a conceptual problem in understanding HPH. This lack of understanding hinders hospitals' ability to effectively reorient health services. A case study of NHS Ayrshire and Arran showed that it was difficult for key staff members to access training [35]. Johansson and other scholars [19] indicate that hospital staff members need to understand the concept of health determinants and to enhance their proficiency in communication. In addition, program evaluation should be included in training agendas [30]. NHS Health Scotland [35] found that a so-called voluntary training program that did not provide a specific time slot for the staff to participate in that program forced the staff to participate using their days off or annual leave to receive such training, which can be a problem. 


\section{Individual Needs and Values}

Individual needs and values refers to explicit psychological factors that generate aspirations and values that drive individual actions or thoughts [5]. Most importantly, the values of individual organisational members should be congruent with the values of the organisation [4]. To ensure that the staff and the organisational values are aligned, staff involvement throughout the HP process becomes crucial. The WHO HPH manual and self-assessment tool (Standard 4.2.5) requires that staff are involved in hospital policymaking, audit and review, while hospitals perform workplace health promotion [9].

\section{Motivation}

Motivation is 'aroused behaviour inclination to approach goals, take essential action, and keep on until fulfillment is attained' [5]. The involvement of the staff in health promotion initiatives is the key to a comprehensive HPH approach [43]. Broadly speaking, the involvement of hospital staff in the process of policy-making, audit and review required by the WHO HPH manual and self-assessment form involves staff needs, and this involvement in turn increases staff motivation.

Regarding barriers to enhancing the motivation of the staff, previous studies found a problematic lack of incentives for hospital staff members to participate in the HPH initiative $[20,50]$. However, it is possible to increase the motivation of hospital staff to participate in the implementation of HPH. In Scotland, it was found that the process of the HPHS initiative could mobilise the participation of the staff in health improvement initiatives [35].

\section{Individual and Organisational Performance}

Burke and Litwin [5] measure individual, group and organisational performance as goal accomplishment, which is usually represented as productivity, customer satisfaction, profit and quality. In the Integration Model, a performance appraisal system and continuing professional development, including health promotion, are required. Johnson [20] find that it is important for hospitals to recognise the efforts of staff members to promote health, which in turn could facilitate the development of HPH. However, Johnson also reports that the approach used in the current performance system is not highly supportive of the development of health promotion in hospitals. Johansson et al. [19] finds that to increase efficiency, there must be a shift in focus from productivity to quality. This shift involves a higher quality of care and more interaction between care providers and caregivers. The author argues that the existing productivity-oriented perspective, which concentrates heavily on efficiency, reduces the efficacy of the health promotion approach.

\section{Conclusion}

This review has reaffirmed the significance of organisational capacity building for health promotion in the development of HPH, has examined and clarified the HPH concept and has highlighted the development of healthpromoting settings in hospitals. This review mainly drew upon the Burke-Litwin Causal Model as a comprehensive framework to understand how the hospital organisation functions and how a 'hospital' can be more efficiently converted into an 'HP hospital'. Based on the framework of the Burke-Litwin Model, this review presented the particular dimensions of organisational capacity that facilitated the development of HPH and the particular parameters of organisational capacity that were required to facilitate the development of HPH.

The review has found that hospitals require systematic organisational support to fulfil their roles in health promotion, including transformational and transactional factors in organisations. In light of empirical studies, facilitating transformational factors include a supportive policy context, external operational support [35], supportive leadership [13, 21, 39, 55], an HP-inclusive mission and strategy [21], making HP a priority [19], a pro-HP culture [19] and an established HP structure [43]. Enabling transactional factors encompass available resources [3, 13], healthy policies [21,36], integration into subsystems [3], effective project management $[36,43]$, the health promotion involvement of the staff [43], the incorporation of health promotion into job descriptions [23] and recognised efforts of staff members on health promotion [20]. Pro-HP culture refers to the holistic and salutogenic view of health rather than illness and emphasises quality rather than only quantity [19]. Available resources cover personnel, funding, allocated time, skilled HP support, access to tools and physical facilities.

However, systematic reviews have indicated that many of the barriers identified are related to insufficient organisational support. There is room for improvement in building organisational capacity to perform health promotion. In light of previous studies, transformational barriers include a lack of government leadership and policy commitment [20,53]; inadequate health insurance coverage of HP [13, 20]; HPH not linked to existing relevant initiatives [35]; a low prioritisation of HP (by leaders) in missions $[20,35,53,54]$; hospital managers with a limited understanding of HPH [13, 30]; a lack of HP background within the hospital [50] and an attitude preferring risk, treatment and quantity to health, prevention and quality [19, 22, 54]. 
The transactional barriers include a lack of clearly determined links between the hospital and community organisations [22]; conflict within multidisciplinary teams, which prevents teams from working together on common issues [20]; a lack of involvement of the directors in the quality management team $[3,10]$; a lack of a specialised department to lead/coordinate [30]; a lack of comprehensive managerial structures to foster HP [21, 54]; shortages of resources, including funds, personnel, time, guidelines, tools, evidence of the effectiveness of health promotion, facilities and physical space [1, 10, 13, 20, 22, 30, 35, 50]; a lack of HP policies [20, 50]; ineffective project management and insufficient planning; inadequate theoretical references; little consideration of underlying structural or cultural problems or of the health of the organisation and the staff; little action for evaluation [1-3, 10, 30]; a lack of communication, coordination and integration $[1,10,30,50$, $54,55]$; invisibility of health promotion [20, 30, 50]; many hospital staff members appearing to be unaware of community services [22]; poor job-person matches; a lack of competence in HP; a lack of proficiency in communication $[10,13,20,32,35,55]$ and a lack of incentives [20, 50]. In particular, low prioritisation of HP in the mission, shortages of resources, ineffective project management, a lack of communication, poor coordination and integration and inappropriate job-person matches were the five crucial barriers. To sustain and improve health promotion outcome, hospitals should consider building a supportive organisation in advance or at least concurrently with the development of the HPH program.

Open Access This article is distributed under the terms of the Creative Commons Attribution License which permits any use, distribution, and reproduction in any medium, provided the original author(s) and the source are credited.

\section{References}

1. Aujoulat I, Le Faou A, Sandrin-Berthon B, Martin F, Deccache A (2001) Implementing health promotion in health care settings: conceptual coherence and policy support. Patient Education Couns 45(4):245-254

2. Bensberg M, Kennedy M (2002) A framework for health promoting emergency departments. Health Promot Int 17(2):179-188

3. Brandt E, Schmidt W, Dziewas R, Groene O (2005) Implementing the Health Promoting Hospitals strategy through a combined application of the EFQM excellence model and the balanced scorecard. In: Groene O, Garcia-Barbero M (eds) Health promotion in hospitals: evidence and quality management. World Health Organization Regional Office for Europe, Copenhagen, pp 80-99

4. Burke WW (2002) Organization change: theory and practice. Sage Publications, Thousand Oaks

5. Burke WW, Litwin GH (1992) A causal model of organizational performance and change. J Manag 18(3):523-545

6. Cummings TG, Worley CG (2009) Organization development and change, 9th edn. South-Western College Publishing, Mason
7. Doherty D (1998) Challenges for hospital policy in Europe- What role can health promotion play? In: Pelikan JM, Krajic K, Lobnig $\mathrm{H}$ (eds) Feasibility, effectiveness, quality and sustainability of health promoting hospital projects. Proceedings of the 5th international conference on Health Promoting Hospitals. Health Promotion Publications, Gamburg, pp 36-41

8. Garcia-Barbero M (1998) Evolution of health care systems. In: Pelikan JM, Krajic K, Lobnig H (eds) Feasibility, effectiveness, quality and sustainability of health promoting hospital projects. Proceedings of the 5th international conference on Health Promoting Hospitals. Health Promotion Publications, Gamburg, pp 24-30

9. Groene O (ed) (2006) Implementing health promotion in hospitals: manual and self -assessment forms. WHO Regional Office for Europe, Copenhagen

10. Groene O, Brandt E, Schmidt W, Moeller J (2009) The balanced scorecard of acute settings: development process, definition of 20 strategic objectives and implementation. Int $\mathbf{J}$ Qual Health Care 21(4):259-271

11. Groene O, Jorgensen JS (2005) Health promotion in hospitals: a strategy to improve quality in health care. Eur J Pub Health 15(1): 6-8

12. Groene O, Jorgensen SJ, Fugleholm AM, Moller L, GarciaBarbero M (2005) Standards for health promotion in hospitals: development and pilot test in nine European countries. Int $\mathbf{J}$ Health Care Qual Assur Inc Leadersh Health Serv 18(4):300-307

13. Guo XH, Tian XY, Pan YS, Yang XH, Wu SY, Wang W, Lin V (2007) Managerial attitudes on the development of Health Promoting Hospitals in Beijing. Health Promot Int 22(3):182-190. doi:10.1093/heapro/dam010

14. Hancock T (1994) Health promotion in Canada: did we win the battle but lose the war? In: Pederson A, O'Neill M, Rootman I (eds) Health promotion in Canada: provincial, national and international perspectives. W.B. Saunders Company Canada, Toronto, pp 350-373

15. Hancock T (1999) Creating health and Health Promoting Hospitals: a worthy challenge for the twenty-first century. Leadersh Health Serv 12(2):8-19

16. Hawe P, Noort M, King L, Jordens C (1997) Multiplying health gains: the critical role of capacity-building within health promotion programs. Health Policy 39(1):29-42

17. Hayes $\mathbf{J}$ (2007) The theory and practice of change management. Palgrave Macmillan, New York

18. Heward S, Hutchins C, Keleher H (2007) Organizational change: key to capacity building and effective health promotion. Health Promot Int 22(2):170-178

19. Johansson H, Weinehall L, Emmelin M (2010) "If we only got a chance". Barriers to and possibilities for a more health-promoting health service. J Multidiscip Healthc 3:1-9

20. Johnson AE (1998) Reorienting a hospital to be more health promoting: a case study of the women's and children's hospital. Adelaide The Flinders University of South Australia, Adelaide

21. Johnson AE, Baum F (2001) Health Promoting Hospitals: a typology of different organizational approaches to health promotion. Health Promot Int 16(3):281-287

22. Johnson AE, Nolan J (2004) Health Promoting Hospitals: gaining an understanding about collaboration. Aust J Prim Health 10(2):51-60. doi:10.1071/PY04026

23. Johnson AE, Paton K (2007) Health promotion and health services: management for change. Oxford University Press, Sydney

24. Johnson JL (2000) The health care institution as a setting for health promotion. In: Poland BD, Green LW, Rootman I (eds) Settings for health promotion: linking theory and practice. Sage Publications, Thousand Oaks, pp 175-198

25. Kotter JP (1978) Organizational dynamics: diagnosis and intervention. Addison-Wesley, Reading 
26. Labonte R (1994) Death of a program, birth of a metaphor: the development of a health promotion in Canada. In: Pederson A, O'Neill M, Rootman I (eds) Health promotion in Canada: provincial, national and international perspectives. W.B. Saunders Company Canada, Toronto, pp 72-90

27. Labonte R, Laverack G (2001) Capacity building in health promotion, Part 1: for whom? And for what purpose? Crit Public Health 11(2):111-127. doi:10.1080/09581590110039838

28. Leavell HR, Clark EG (1965) Preventive medicine for the doctor in his community: an epidemiologic approach. McGraw-Hill, New York

29. Leavitt HJ (1965) Applied organizational change in industry. In: March JG (ed) Handbook of organizations. Rand McNally, New York, pp 1144-1170

30. Lin YW, Huang HL, Tung SC (2009) The organisational diagnosis of a health promoting hospital in Taiwan. Patient Education Couns 76(2):248-253. doi:10.1016/j.pec.2008.12.025

31. Lin YW, Lin YY (2010) Health-promoting organization and organizational effectiveness of health promotion in hospitals: a national cross-sectional survey in Taiwan. Health Promot Int 26(3):362-375

32. McBride A (2004) Health promotion in the acute hospital setting: the receptivity of adult in-patients. Patient Education Couns 54(1):73-78

33. Mchugh C, Robinson A, Chesters J (2010) Health promoting health services: a review of the evidence. Health Promot Int 25(2):230-237

34. Nadler DA, Tushman ML (1977) A diagnostic model for organization behavior in organizations. In: Hackman JR, Lawler EE III, Porter LW (eds) Perspectives in behavior in organizations. McGraw-Hill, New York

35. NHS Health Scotland (2010) Review of health promoting health service funded sites. NHS Health Scotland, Edinburgh

36. Nowak P, Lobnig H, Krajic K, Pelikan JM (1998) Case study Rudolfstiftung Hospital, Vienna, Austria-WHO-model project, health and hospital. In: Pelikan JM, Garcia-Barbero M, Lobnig H, Krajic K (eds) Pathways to a health promoting hospital. Experiences from the European Pilot Hospital Project 1993-1997. Health Promotion Publications, Gamburg, pp 47-66

37. Nowak P, März R (1998) The Vienna WHO-Model project "health and hospital". In: Pelikan JM, Krajic K, Lobnig H (eds) Feasibility, effectiveness, quality and sustainability of health promoting hospital projects. Proceedings of the 5th international conference on Health Promoting Hospitals. Health Promotion Publications, Gamburg, pp 164-169

38. Põlluste K, Alop J, Groene O, Härm T, Merisalu E, Suurorg L (2007) Health-promoting hospitals in Estonia: what are they doing differently? Health Promot Int 22(4):327-336

39. Põlluste K, Härm T, Merisalu E, Suurorg L (2006) Development of the HPH network in Estonia: the managers' perspective. Health Promot Hosp Newsl 26:5-7

40. Pelikan JM (2007) Health Promoting Hospitals-assessing developments in the network. Italian J Public Health 5(4):261-270

41. Pelikan JM (2007) Understanding differentiation of health in late modernity by use of sociological systems theory. In: Kickbusch I, McQueen DV (eds) Health and modernity: the role of theory in health promotion. Springer, New York, pp 74-102

42. Pelikan JM (2010) Towards a model for health development or promotion in hospital settings. 20th IUHPE World Conference, Geneva, 15 July 2010

43. Pelikan JM, Dietscher C, Krajic K, Nowak P, Brandt E, Favaretti C, Garel P, Groene O, Güntert BJ, Kerr A, Tschumy EM, McCartney R, Tountas Y (2006) Putting HPH policy into action
WHO collaborating centre for health promoting in hospitals and health care, Vienna

44. Pelikan JM, Dietscher C, Schmied H (2012) What organizational capacities do make a difference for the implementation of health promotion in HPH hospitals? 20th international conference on HPH Taipei, Taiwan 12-13 April 2012

45. Pelikan JM, Groene O, Svane JK (2011) The international HPH network-a short history of two decades of development. Clin Health Promot 1(1):32-36

46. Pelikan JM, Krajic K, Dietscher C (2001) The health promoting hospital (HPH): concept and development. Patient Education Couns 45(4):239-243

47. Pelikan JM, Lobnig H, Krajic K (1997) Health-promoting hospitals. World Health 50(3):24-25

48. Tønnesen H, Nielsen P, Lauritzen J, Moller A (2009) Smoking and alcohol intervention before surgery: evidence for best practice. Br J Anaesth 102(3):297

49. Tichy NM (1983) Managing strategic change: technical, political, and cultural dynamics. Wiley, New York

50. Tountas Y, Pavi E, Tsamandouraki K, Arkadopoulos N, Triantafyllou D (2004) Evaluation of the participation of Aretaieion hospital, Greece in the WHO pilot project of Health Promoting Hospitals. Health Promot Int 19(4):453-462

51. Wang YW (2008) The international development of health promotion. J Healthc Qual 2(3):4-9

52. Weisbord MR (1976) Organizational diagnosis: six places to look for trouble with or without a theory. Group Organ Manag 1(4): $430-447$

53. Whitehead D (2004) The European Health Promoting Hospitals (HPH) project: how far on? Health Promot Int 19(2):259-267

54. Whitelaw S, Graham N, Black D, Coburn J, Renwick L (2011) Developing capacity and achieving sustainable implementation in healthy 'settings': insights from NHS Health Scotland's health promoting health service project. Health Promot Int 27(1):127-137. doi:10.1093/heapro/dar038

55. Whitelaw S, Martin C, Kerr A, Wimbush E (2006) An evaluation of the health promoting health service framework: the implementation of a settings based approach within the NHS in Scotland. Health Promot Int 21(2):136-144

56. WHO-EURO (2001) Seventh workshop of National/Regional Health Promoting Hospitals network coordinators. National/ Regional Health Promoting Hospitals network coordinators. World Health Organization-Regional Office for Europe, Copenhagen

57. WHO-EURO (2007) The international network of Health Promoting Hospitals and health services: integrating health promotion into hospitals and health services. World Health WorldRegional Office for Europe, Copenhagen

58. WHO-EURO (2009) The European health report 2009: health and health system. World Health Organisation Copenhagen

59. WHO (1978) Alma-Ata 1978: primary health care, health for all, Series No. 1 World Health Organisation, Geneva

60. WHO (1986) The Ottawa charter for health promotion. World Health Organization, Geneva

61. WHO (2009) 7th Global Conference on Health Promotion: Nairobi 2009. World Health Organization, Geneva

62. Wise M, Nutbeam D (2007) Enabling health systems transformation: what progress has been made in re-orienting health services? Promot Education 14(2 suppl):23-27. doi:10.1177/ $10253823070140020801 x$

63. Yeatman HR, Nove T (2002) Reorienting health services with capacity building: a case study of the core skills in health promotion project. Health Promot Int 17(4):341-350. doi:10.1093/ heapro/17.4.341 